\title{
A new hope: the immunotherapy in small cell lung cancer
}

\author{
Minireview \\ Q. $\mathrm{LI}^{1}$, D. YUAN ${ }^{1}$, C. MA 1 , Y. $\mathrm{LIU}^{2}$, L. MA ${ }^{1}$, T. $\mathrm{LV}^{1}$, Y. SONG ${ }^{1, *}$ \\ ${ }^{1}$ Department of Respiratory and Critical Care Medicine, Jinling Hospital, School of Medicine, Nanjing University, Nanjing, China; ${ }^{2}$ Department \\ of Respiratory Medicine, Jinling Hospital, Southern Medical University (Guangzhou), Nanjing, China
}

${ }^{*}$ Correspondence: yong_song6310@yahoo.com

Received October 1, 2015 / Accepted February 1, 2016

\begin{abstract}
Small cell lung cancer (SCLC) is of a high-grade malignancy with a high metastatic potential and poor clinical prognosis. Unfortunately, SCLC initially exhibits a good response to chemotherapy and radiation therapy, but inevitably, relapses decrease patients' chance of survival. Despite tremendous advances on the development of new chemotherapeutic agents, the prognosis of this disease remains poor. Immunotherapy plays a role in eliciting an anticancer response by modulating the patient's immune response of the tumor. Several studies have demonstrated that abnormal autoimmune regulation has a close relationship with SCLC. Thus, several immunotherapy trials are focused on SCLC treatment, including such approaches as immune checkpoints blockers, tumor vaccine, antigenic targets and adoptive cellular immunotherapy to benefit patients with SCLC. To date, the results from immunotherapy in SCLC have not been promising. For example, tumor vaccines have not been demonstrated to have a significant survival benefit. However, there have been many promising advances with immune checkpoints blockers. This review will provide a general overview of immunotherapy in SCLC. The landmark clinical trials in previous successful immunotherapy studies are summarized here. Finally, the challenges of immunotherapy in SCLC are discussed to facilitate the prediction of possible and valuable strategies for future therapy.
\end{abstract}

Key words: small cell lung cancer (SCLC), immunotherapy, combination therapy, immune checkpoints, tumor vaccine

Lung cancer remains the leading cause of cancer-related deaths worldwide [1]. Lung cancer is classified into non-small cell lung cancer (NSCLC) and small cell lung cancer (SCLC). SCLC, a histological subtype, accounts for approximately $15 \%$ of all lung cancers cases. SCLC patients are classified into limited disease SCLC (LD-SCLC) and extensive disease SCLC (ED-SCLC). For LD-SCLC, chemotherapy (cisplatin or carboplatin with etoposide) combined with thoracic radiotherapy is the standard strategy [2]. Systemic chemotherapy is recommended for the treatment of patients with limited and extensive SCLC. Unfortunately, SCLC initially shows a good response to chemotherapy and radiation therapy, but relapses result in poor survival. The median survival of this patient group is approximately $7-12$ months $[3,4]$. Although new chemotherapeutic agents are being continuously developed, the prognosis of this disease remains poor due to limited treatment efficacy $[5,6]$. Traditional therapies (radiotherapy and chemotherapy) have limited effectiveness; therefore, new strategies to improve outcome are urgently needed.

Targeted molecular therapies such as Erlotinib and Crizotinib have brought significant advances in the treatment of NSCLC. However, there has been limited success with these targeted approaches in clinical trials for SCLC [7]. Although many researchers have studied the efficacy of targeted molecular therapy for SCLC, unfortunately, the results from clinical studies of investigational new drugs are not encouraging due to the tumor heterogeneity at the genetic level [8]. This leads to the question of whether any successful strategies can be developed for SCLC.

Immunotherapy could help solve this problem. Immunotherapy plays a role in eliciting an anticancer response by modulating the patient's immune response to the tumor, 
specifically by enhancing host immune surveillance and/or decreasing tolerance to tumor cells [9]. Recent studies have demonstrated that immunotherapy achieves a good response against tumor cells in lung cancer. Numerous immunotherapy trials are focused on NSCLC treatment, but few studies have been conducted to examine the efficacy of immunotherapy for the treatment of SCLC.

Previous studies have indicated that abnormal autoimmunity regulation is closely related to SCLC. SCLC patients with immune-mediated Lambert-Eaton myasthenic syndrome are prone to remain in a limited stage for a longer period of time and have improved survival [10]. In addition, patients with higher ratios of forkhead box protein P3 (FOXP3)expressing cells infiltrating into tumors have a worse survival rate [11]. Furthermore, a higher ratio of antitumor effector $\mathrm{T}$ cells (Teffs) to regulatory $\mathrm{T}$ cells was observed in longterm survivors of SCLC compared with those who had recurrent disease [12]. An analysis of T cells in peripheral blood samples also reveals higher counts of immune Teffs in LD-SCLC patients than in ED-SCLC patients [13]. These observations suggest that strategies such as shifting the Tcell balance that act by promoting antitumor immunity can delay tumor growth in patients with SCLC. There has been notable progress in SCLC immunotherapy-based therapeutic approaches such as immune checkpoints, tumor vaccine and antigenic targets. This article reviews recent clinical trials in SCLC treatments that utilize immunology to induce antitumor effects (Table 1).

\section{Immune checkpoint inhibitor}

A series of regulatory pathways maintain a balance between the appropriate recognition and destruction of pathogens or tumors to prevent the overstimulation of immune responses. Costimulatory and co-inhibitory factors play a key role in immune regulation after stimulation of the T-cell receptor by fine-tuning the antigen-specific T-cell response [14]. The first step in the generation of a specific T-cell response is that the $\mathrm{T}$-cell receptor (TCR) recognizes the antigenic peptides in the context of major histocompatibility complex (MHC) molecules on the surface of antigen-presenting cells (APCs) [15]. However, the entire T-cell activation process needs a second "costimulatory" signal as well as TCR-antigenic-MHC. This second signal is generated during the costimulatory receptor$\mathrm{CD} 28$ on the surface of $\mathrm{T}$ cells binding to $\mathrm{B} 7$ ligand subtypes CD80 and CD86 on the APC. There are other similar types of molecules including CD134, and CD137 that enhance the signal that leads to T-cell activation and thereby help to potentiate the immune response by co-stimulation through CD28. Co-inhibition molecules such as cytotoxic T-lymphocyteassociated antigen-4 (CTLA-4), programmed death-1 (PD-1), $\mathrm{B} 7-\mathrm{H} 3$, and $\mathrm{B} 7 \mathrm{x}$ weaken antigen-specific immune responses

Table 1. Summary of completed and ongoing immunotherapy trials in small cell lung cancer.

\begin{tabular}{|c|c|c|c|c|c|c|}
\hline Target & Ref & Immunotherapy & Stage & Phase & No. & Results \\
\hline \multicolumn{7}{|c|}{ Immune checkpoint inhibitor } \\
\hline \multirow[t]{2}{*}{ PD-1 } & {$[24]$} & nivolumab vs nivolumab+ipilimumab & $\mathrm{I} / \mathrm{II}$ & $\begin{array}{l}\text { Recurrent } \\
\text { SCLC }\end{array}$ & 75 & ORR (15\% vs $25 \%)$ \\
\hline & {$[25]$} & pembrolizumab & $\mathrm{Ib}$ & ED-SCLC & 135 & DCR $31 \%$ \\
\hline CTLA-4 & {$[28]$} & $\begin{array}{l}\text { paclitaxel /carboplatin with placebo } \\
\text { (control) or ipilimumab (concurrent } \\
\text { ipilimumab or phased ipilimumab) }\end{array}$ & II & ED-SCLC & 130 & $\begin{array}{l}\text { mPFS of } 5.2,3.9 \text { and } 5.2 \text { months; mOS of } \\
9.9,9.1 \text { and } 12.9 \text { months. }\end{array}$ \\
\hline \multicolumn{7}{|c|}{ Adoptive cellular immunotherapy } \\
\hline T lymphocyte & {$[30]$} & EAAL & - & SCLC & 32 & $\begin{array}{l}\text { OS (17.6 vs } 10 \text { months, } \mathrm{P}=0.060, \mathrm{HR}=0.487 \text {, } \\
95 \% \text { CI } 0.228 \sim 1.037)\end{array}$ \\
\hline CIT & {$[31]$} & $\mathrm{NK}, \gamma \delta \mathrm{T}$, and CIK cell & - & SCLC & 58 & $\begin{array}{l}\text { LD-SCLC: OS } \\
(26.5 \text { vs. } 11.8 \text { months, } \mathrm{P}=0.033 ; \mathrm{HR}, 0.405 \text {, } \\
95 \% \text { CI, } 0.169-0.972, \mathrm{P}=0.043)\end{array}$ \\
\hline \multicolumn{7}{|l|}{ Tumor vaccine } \\
\hline \multirow[t]{2}{*}{ BEC2/BCG vaccine } & {$[34]$} & BEC2 plus BCG & - & SCLC & 15 & $\begin{array}{l}\text { mRRS for patients with ED-SCLC was } 11 \\
\text { months and LD-SCLC was }>47 \text { months. }\end{array}$ \\
\hline & {$[35]$} & $\mathrm{BEC} 2$ plus BSC & III & LD-SCLC & 515 & OS (16.4 vs 14.3 months) \\
\hline \multirow[t]{2}{*}{ INGN-225 } & {$[37]$} & Ad.p53-DC & - & ED-SCLC & 29 & ORR to chemotherapy $61.9 \%$. \\
\hline & {$[38]$} & Ad.p53-DC & $\mathrm{I} / \mathrm{II}$ & ED-SCLC & 54 & $\begin{array}{l}\text { Specific anti-p53 immune response was } \\
18 / 43(41.8 \%) \text {, overall post-INGN-225 } \\
\text { response was } 17 / 33(51.5 \%)\end{array}$ \\
\hline
\end{tabular}


by limiting their magnitude and duration. These co- inhibition molecules are called "immune checkpoint proteins." Immune checkpoint inhibition has showed promising advances in cancer immunotherapy, including blockade of CTLA-4 and programmed death receptor ligand (PD-L1) (Figure 1). Blockade of immune checkpoints with monoclonal antibodies is considered as a promising therapeutic tool in oncology [16], including SCLC. A study investigating CTLA-4, PD-1 and PD-L1 distribution and clinical value in liquid biopsy (such as blood) of SCLC patients showed that those cytokines are not only key components of immune checkpoints for cancer, but they are also highly expressed in liquid biopsy of SCLC patients [17].

PD-1 receptor blockade. PD-1 belongs to the CD28 family of proteins. It is a receptor located on the surface of $\mathrm{T}$ cells to regulate their proliferation and activation [18]. Its ligand is programmed cell death-ligand 1 (PD-L1), a $40-\mathrm{kDa}$ type 1 transmembrane protein. PD-L1 can suppress the immune system in cases of autoimmune disease and viral infections. The formation of the PD-1/PD-L1 pathway can transmit an inhibitory signal to reduce $T$ cell activity followed by suppression of the immune system. PD-1 receptor-ligand interaction is often hijacked by tumors to perturb immune-tolerance or negatively control antigen-specific T-cell activity [19]. Inhibition of this pathway with anti-PD-1/PD-L1 antibodies has been demonstrated to exert a promising antitumor effect against several human malignancies, including NSCLC [20, 21]. Is this type of effectiveness applicable to SCLC? In vitro, PD-1 and PD-L1 molecules are co-expressed on the surface of SCLC cells. Studies have implied that PD-1 and its ligand on the SCLC cells can participate in the growth inhibition of tumor cells as reported in cytotoxic $\mathrm{T}$ cells [22]. In vivo, the expression of PD-L1 in tumor cells was significantly correlated with LD-SCLC, and it showed significantly longer overall survival (OS) than PD-L1-negative patients. This seems to be contradictory to the rationales of using PD-1/PD-L1 mAbs/ blockers in clinical trails, as the PD-1/PD-L1 pathway inhibits anti-cancer immunity in most cases. This could be due to the expression of PD-L1 being significantly higher in SCLC patients with LD-SCLC than in those with ED-SCLC. Given that a LD stage has been reported to be a prognostic factor in SCLC patients, PD-L1 expression has been related to a good prognosis. Additionally, the threshold for positivity not being clearly defined could contibute to the inconclusive results [23]. A few clinical trials have attempted to explore the efficacy of the PD-1 receptor blockade in SCLC.

Nivolumab is a fully human IgG4 PD-1 immune checkpoint inhibitor. A phase I/II study mainly investigated whether combined blockade of PD-1 and CTLA- 4 immune checkpoint pathways could improve the anti-tumor activity with a manageable safety profile in SCLC [24]. Key eligibility criteria for the SCLC cohort include: confirmed, measurable disease, and failure of standard therapy. Seventy-five patients were enrolled. The patients were randomized assigned to the Nivolumab group ( $3 \mathrm{mg} / \mathrm{kg}$, intravenous injection, two times per week) group or the Nivolumab + Ipilimumab group $(1+1 \mathrm{mg} / \mathrm{kg}, 1+3 \mathrm{mg} / \mathrm{kg}$ or $3+1 \mathrm{mg} / \mathrm{kg}$; intravenous injection; three times per week for 4 cycles followed by Nivolumab $3 \mathrm{mg} / \mathrm{kg}$ two times per week). The number of patients in the Nivolumab group was 40 , and the Nivolumab + Ipilimumab group included 35 patients. In the Nivolumab group, 6 (15\%) experienced partial response (PR), 9 (22.5\%) experienced stable disease (SD) and 25 (62.5\%) experienced progressive disease (PD). In 20 evaluable Nivolumab + Ipilimumab patients, 1 (5\%) had complete response (CR), 4 (20\%) had a PR, $6(30 \%)$ had SD and $9(45 \%)$ had PD. ORR was $15 \%$ (Nivolumab) and 25\% (Nivolumab + Ipilimumab) for the evaluable patients, respectively. The responses were durable and were presented independent of initial platinum sensitive or refractory, and they were as not dependent on PD-L1 expression. Adverse events were tolerable. Drug-related adverse events occurring in $\geq 10 \%$ of the patients were fatigue (18\%), diarrhea $(13 \%)$, nausea (10\%), and decreased appetite (10\%) in the Nivolumab group; while fatigue (29\%), diarrhea (17\%), pruritus (14\%), nausea, endocrine disorders and rash (11\% each) occurred in the Nivolumab + Ipilimumab group. In summary, Nivolumab + Ipilimumab has the potential to cure SCLC. Safety and biomarker analysis will be presented along with new clinical activity.

Pembrolizumab is an anti-PD-1 monoclonal antibody that is designed to block the PD-1/PD-L1 pathway. The treatment options for patients with metastatic SCLC who are on platinum-based chemotherapy are limited. In this context, a phase Ib study was launched to assess the safety and efficacy of Pembrolizumab in patients with PD-L1+ SCLC. Of the 135 patients with SCLC who were screened, 37 (27\%) had PD-L+ tumors. Sixteen patients received Pembrolizumab after platinum and etoposide. Four of the 16 (25\%) evaluable patients underwent a PR. One (7\%) patient had SD. The disease control rate is approximately $31 \%$. Nine patients experienced drugrelated adverse events; only one patient had a degree of grade $\geq 3$ [25]. Pembrolizumab has promising antitumor activity in patients with PD-L1+ SCLC who have progressed on prior platinum-based therapy. The safety and efficacy of Pembrolizumab in patients with PD-L1+ SCLC were similar to other malignancies. NCT02359019 and NCT02403920 that investigate pembrolizumab + chemotherapy or pembrolizumab + chemotherapy + radiotherapy in SCLC are ongoing. The results of these correlation studies are expected to be beneficial for the SCLC patients with limited life-span.

Anticytotoxic T-lymphocyte-associated antigen-4. CTLA-4 is another important immune checkpoint protein. It is expressed on activated $\mathrm{T}$ cells, and it has a higher affinity for B7 ligands compared with CD28, thereby competitively inhibiting CD28-mediated T-cell activation and limiting the subsequent T-cell response [26, 27]. Therefore, blockade of the CTLA-4 checkpoint pathway could be a reasonable approach to combat cancer.

Ipilimumab, an anti-CTLA-4 monoclonal antibody, has been approved for treatment of unresectable or metastatic 
melanoma by the Food and Drug Administration (FDA). Can Ipilimumab demonstrate a survival benefit in SCLC? A phase II study evaluated the efficacy of Ipilimumab + Paclitaxel (Taxol)/Carboplatin in ED-SCLC [28]. Patients $(\mathrm{n}=130)$ with chemotherapy-naive ED-SCLC were randomized 1: 1: 1 to receive Paclitaxel /Carboplatin with either placebo (control) or Ipilimumab $10 \mathrm{mg} / \mathrm{kg}$ in two alternative regimens, concurrent with Ipilimumab (Ipilimumab + Paclitaxel/Carboplatin followed by placebo + Paclitaxel/Carboplatin) or phased Ipilimumab (placebo + Paclitaxel/Carboplatin followed by Ipilimumab + Paclitaxel/Carboplatin). Treatment was administered every 3 weeks for a maximum of 18 weeks (induction), followed by maintenance with Ipilimumab or placebo every 12 weeks. Unfortunately, no improvement in PFS (hazard ratio $(\mathrm{HR})=0.93 ; P=0.37)$ or OS $(\mathrm{HR}=0.75 ; P=0.13) \mathrm{oc}-$ curred for the phased Ipilimumab, but immune-related PFS versus control ( $\mathrm{HR}=0.64 ; P=0.03$ ) was improved. While phased Ipilimumab, concurrent Ipilimumab and control were associated with median immune-related PFS of 6.4, 5.7 and 5.3 months, respectively, the median PFS was 5.2, 3.9 and 5.2 months, and the median OS was 12.9, 9.1 and 9.9 months. Ipilimumab combined with Paclitaxel/Carboplatin shows clinical activity in patients with previously untreated EDSCLC when administered as a phased regimen. These results suggest further investigation of Ipilimumab in ED-SCLC.

\section{Adoptive cellular immunotherapy}

Adoptive cellular immunotherapy has been considered as an important antitumor treatment for many years. Various proliferated effector cells, such as lymphokine activated killer (LAK) cells, tumor-infiltrating lymphocytes (TILs), dendritic cells (DCs), activated natural killer (NK) cells and cytokine induced killer (CIK) cells, have shown some anti-tumor effects (Figure 1).

Mediating antigen-specific activation of effector T cells is a critical part of the adaptive immune system. Activation of effector T cells targets tumor cells directly or mediates subsequent humoral antitumor responses. After T-cell activation in response to a specific antigen, a subset of lymphocytes called regulatory $\mathrm{T}$ cells subsequently plays a major role in downregulating the resulting immune response and establishing immune tolerance to the antigens, thereby allowing the body to suppress autoimmunity. Transfusion of an adequate quantity of lymphocytes to recognize and lyse tumor cells was the basis for successful adoptive cell therapy [29].

Expanded activated autologous lymphocytes (EAAL) are T lymphocytes that are isolated from cancer patients with immobilized anti-CD3 monoclonal antibody. It was obtained by proliferation of peripheral blood mononuclear cells (PBMCs) of patients followed by phenotype determination. To investigate the clinical efficacy of EAAL in patients with SCLC, a total of 32 SCLC patients were selected and randomly divided into EAAL treatment and control groups with 16 cases in each group. The OS of the EAAL treatment group was longer than that of control group, but the difference was not statistically significant ( 17.6 vs. 10 months, $P=0.060, \mathrm{HR}=0.487,95 \% \mathrm{CI}$ $0.228 \sim 1.037)$. Although the 1- to 3 -year survival rates in the EAAL treatment group were longer than those in the control group, there was no significant difference $(p>0.05)$ [30]. Generally, EAAL adoptive immunotherapy could prolong the OS of SCLC patients.

In addition to EAAL, cellular immunotherapy (CIT) with autologous natural killer (NK), $\gamma \delta$ T, and CIK cells has also been investigated [31]. A pilot prospective cohort study was conducted with SCLC patients who responded to initial chemotherapy to survey the efficacy and safety of CIT as maintenance therapy for SCLC patients. A total of 58 patients were randomly assigned to receive either CIT as maintenance therapy (study group), or to be followed-up without further treatment (control group). The results showed PFS was not significantly different between the groups, but OS was significantly longer in the study group than the control group (20 vs. 11.5 months, $P=0.005$ ). Correspondingly, OS was longer in the study group compared to the control group (26.5 vs. 11.8 months, $P=0.033$ ) among patients with limited-stage disease. Of particular interest was that PFS and OS were longer in the study group than the control group ( 5 vs. 2.7 months, $P=$ 0.037 ; and 14.5 vs. 9 months, $P=0.038$, respectively) among patients with extensive-stage disease. No significant adverse events occurred in patients undergoing CIT. Thus, integrating CIT into current treatment could be a novel strategy for SCLC therapy.

\section{Cancer vaccine}

Cancer vaccines are designed to present a unique allogeneic tumor antigen, including limited to whole tumor cells, DNA bearing viral vectors, proteins, or peptides [32], to the adaptive immune system, thereby stimulating the humoral immune response against the specific antigen (Figure 1). The ultimate goals of vaccine therapy are producing long-lasting, active memory, which would consequently yield faster and more robust responses to re-exposure. Thus far, the effectiveness of lung cancer vaccine has been discouraging [33]; however, there have been several promising advances in the treatment of SCLC.

BEC2/BCG vaccine. The glycosphingolipid antigen GD3 is highly expressed in SCLC, but it is rarely expressed in normal tissues. BEC2, a monoclonal antibody that mimics GD3 promoted anti-tumor immune by selectively targeting ganglioside GD3. A clinical trial of 15 patients who had completed standard therapy for SCLC received a series of intradermal immunizations consisting of $2.5 \mathrm{mg}$ of BEC2 plus BCG over a 10 -week period [34]. Five patients were determined to have anti-GD3 antibodies, including those with the longest relapsefree survival. The median relapse-free survival for patients with extensive stage disease was 11 months and limited stage disease was $>47$ months. BEC2 plus BCG can induce anti-GD3 antibodies, and it is safe for patients with SCLC after standard 


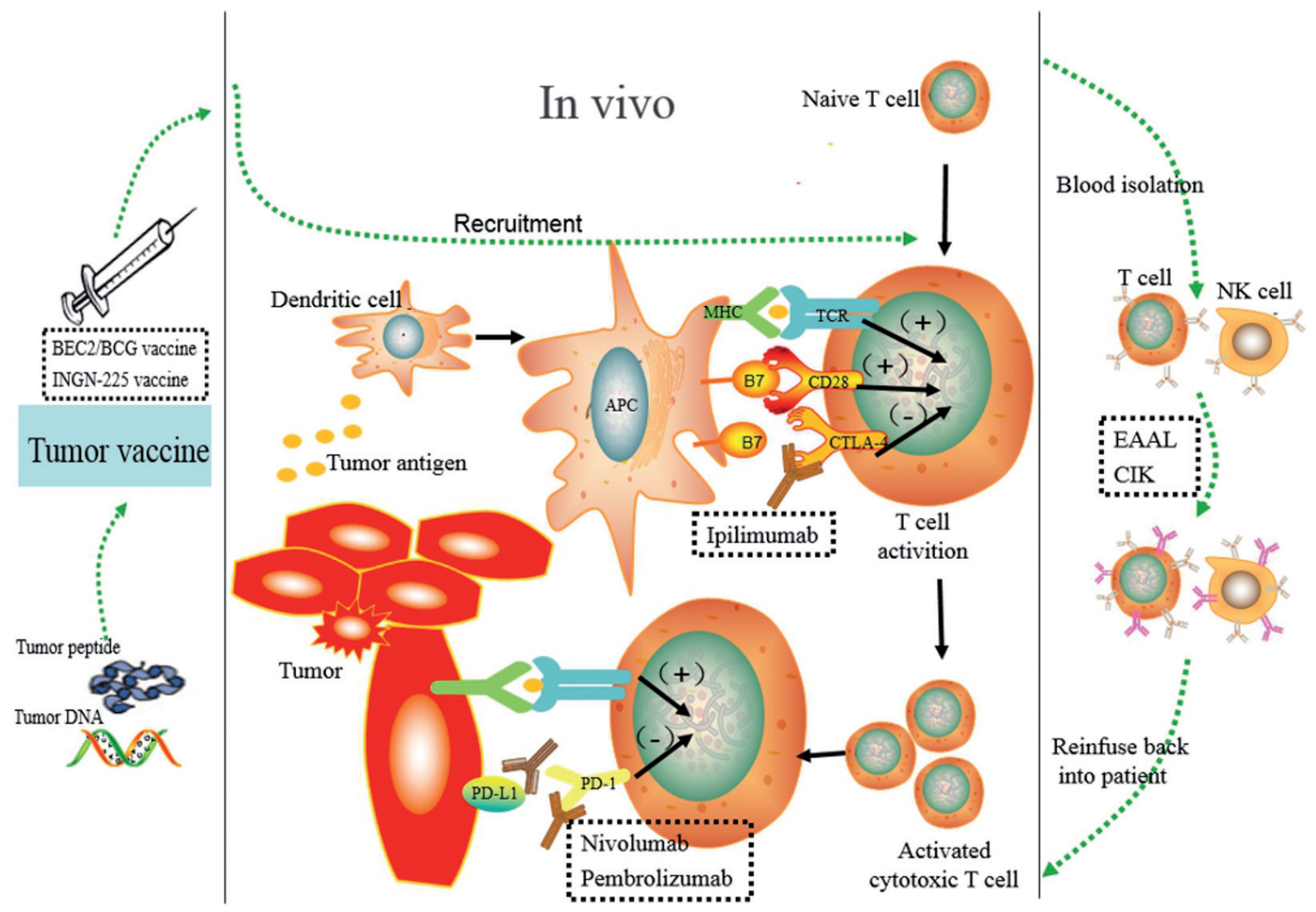

Figure 1. Current immunotherapeutic strategies for small cell lung cancer.

TCR recognizes the antigenic peptides in the context of MHC molecules on the surface of APCs, then the whole T-cell is activated with the help of a second "costimulatory signal" such as B7/CD28. Co-inhibition involving molecules like CTLA-4, PD-1, B7-H3, and B7x weakens antigen-specific immune responses by limiting their magnitude and duration correspondingly. Blockade of immune checkpoints with monoclonal antibodies (Nivolumab, Pembrolizumab, Ipilimumab) can benefit T activation. Various proliferated effector cells, such as TILs, DCs, NK and CIK, can be isolated from cancer patients and followed antigen modification to mediate antigen-specific activation of effector $T$ cells. Cancer vaccines are designed to present a unique allogeneic tumor antigen, including limited to whole tumor cells, DNA bearing viral vectors, proteins, or peptides, to the adaptive immune system, thereby stimulating humoral immune response against the specific antigen.

Abbreviations: APC - antigen-presenting cells; CIK - cytokine induced killer cells; CTLA-4 - cytotoxic T-lymphocyte-associated antigen-4; DC - dendritic cells; EAAL - expanded activated autologous lymphocyte; MHC - major histocompatibility complex; NK - natural killer cells; PD-1 - programmed death-1; PD-L1 - programmed death receptor ligand; TCR - T-cell receptor; TILs - tumor-infiltrating lymphocytes.

therapy. The survival and relapse-free survival in this group of patients was substantially longer than those observed in a prior group of similar patients.

As a result of the impressive survival achievedin the small group of patients with SCLC, a large, randomized phase III trial was initiated for patients with LD-SCLC and complete or partial response to at least 4 cycles of 2 -drug induction chemotherapy and chest radiotherapy [35]. A total of 515 patients with limited-stage SCLC were randomly assigned to receive $\mathrm{BEC} 2 / \mathrm{BCG}$ or BSC. At the end of monitoring interval, the median OS was not significantly different between the groups (16.4 vs. 14.3 months). The PFS also failed to achieve statistical significance. Bec2/BCG vaccination as maintenance therapy in responding patients with limited-disease SCLC is essentially not effective. Bec2/BCG vaccination is one single antigenic target with higher titers of anti-GD3, but additional antigens could be required to formulate a multivalent vaccine.

INGN-225. Over $90 \%$ of SCLC patients harbored p 53 gene mutation, and the $\mathrm{p} 53$ protein has been suggested as a potential antigenic target that can be exploited with immunotherapeutic strategies [36]. INGN-225 was designed for the p53 protein. It is produced from autologous peripheral blood mononuclear cells, and then it is cultured in the presence of IL-4 and granulocyte macrophage colony-stimulating factor prior to incubation with a viral construct containing wild-type p53.

A study was launched to test the immunologic and clinical effects of a new cancer vaccine consisting of DC transduced with the full-length wild-type p53 gene delivered via an ad- 
enoviral vector in patients with ED-SCLC [37]. Twenty-nine patients were vaccinated repeatedly at 2-week intervals. P53specific $\mathrm{T}$ cell responses to vaccination were observed in $57.1 \%$ of the patients. Vaccination-related immunologic responses were positively associated with a moderate increase in the titer of anti-adenovirus antibodies, and negatively with an accumulation of immature myeloid cells. Additionally, a high rate of objective clinical responses to chemotherapy (61.9\%) that immediately followed vaccination was observed. Clinical response to subsequent chemotherapy was closely associated with induction of immunologic response to vaccination. This study provides clinical support for an emerging paradigm in cancer immunotherapy wherein the optimal use of vaccination could be more effective, not as a separate modality, but in direct combination with chemotherapy. Subsequently, the updated study that enrolled 54 patients with ED-SCLC revealed that most of the patients had disease progression (two patients (3.7\%) had a PR, and 13 achieved SD that was specifically related to the vaccine).

A more recent phase I/II study [38] explored INGN-225's role in SCLC treatment. Patients were then randomized 1:1:1 to arm A (standard of care), arm B (INGN-225 only), or arm C (INGN-225 plus ATRA). Post-INGN-225 response was observed in 11/14 (78.6\%) and 5/15 (33\%) patients with positive and negative immune responses, respectively. INGN-225 was well tolerated (all toxicities $\leq$ grade 2 ) in the Phase I/II trial (54 patients receiving at least 1 dose). Specific anti-p53 immune response was positive in $18 / 43(41.8 \%)$ patients, with overall post-INGN-225 response observed in 17/33 (51.5\%), and immune response data are available for 29 patients (14 positive, 15 negative). INGN-225 is safe, induces a significant immune response, and appears to sensitize SCLC to subsequent chemotherapy. Improvements in immune response induction and understanding the chemotherapy-immunotherapy synergism will determine INGN-225's future role as an anticancer therapy.

The tumor microenvironment has been shown to interfere with effective immune recognition [39]. There is growing evidence to suggest that the elimination of myeloid-derived suppressor cells (MDSCs) during medical interventions can improve the prognosis of SCLC patients [40]. In a clinical trial of patients with ED-SCLC [41], forty-one patients were randomized into three arms: arm A- control, arm B- vaccination with dendritic cells transduced with wild-type p53, and arm C- vaccination in combination with MDSC targeted therapy with all-trans retinoic acid (ATRA). MDSC depletion in cancer patients can improve the antitumor immune response to vaccination, suggesting that cancer vaccine combination targeting the tumor microenvironment factors could be an ideal strategy.

Several factors including reduced antigen presentation, antigenic loss, cytokines, immunosuppressive cells and immune checkpoints have been recognized to associate with tumor escape [42]. Vaccine-induced T-cell responses to overcome the tumoral mechanisms of immune escape could lack significant clinically outcomes; however, cancer vaccines continue to be studied as adjunctive therapy.

\section{Others}

Inhibition that potentially synergizes with cytotoxic and targeted therapeutics could be beneficial for SCLC patients. An open-label, randomized phase II study (NCT01439568) showed that selected ED-SCLC patients with high levels of circulating tumor cells (CTCs) and/or CXCR4 expression on CTCs and/or tumor tissue at baseline can benefit by the addition of CXCR4 antagonist LY2510924 (LY) based on results from receiver operating characteristic curves [43].

\section{Challenges in immunotherapy for SCLC}

There are many challenges in developing immunotherapy for SCLC. First, the biology of SCLC is complex and has not been fully elucidated. The complex genetic heterogeneity observed in SCLC patients limits the success of any treatment, including immunotherapy. SCLC evades the immune response to tumors more effectively by greatly reducing the transcription of HLA-A, B, and C and beta $2 \mathrm{~m}$ genes compared with other lung cancer types [44]. Immune escape is closely associated with the recurrence of the disease leading to poor patient survival [45]. Several studies have indicated that the immune system can promote the control of cancer in concert with conventional chemotherapy or radiotherapy. Cell death due to radiotherapy and some chemotherapeutic agents can induce immune-stimulatory effects by encouraging tumor antigen-specific immune responses or by altering the immunologic properties of any remaining tumor cells. This anticancer immune response then helps to eliminate residual cancer cells that survived chemotherapy or radiotherapy or maintain micro-metastases in a stage of dormancy [46]. This phenomenon, called the "abscopal effect" was initially hypothesized to indicate that radiation provides an antigen release that stimulates effector immune cells to induce tumor cell death outside of the irradiation field [47]. This is an indication that the schedule of immunotherapy in relation to other therapies can result in the best benefits for patients with SCLC. Secondly, there are no prognostic or predictive biomarkers in SCLC. No indication can be used to select SCLC patient subgroups that could benefit the most from specific immunotherapeutic approaches. Yet, identifying patient populations that can derive the greatest benefit to treatment remains a therapeutic challenge. In addition, response to immunotherapy requires time. SCLC is a high-grade malignancy, and it possesses a high tendency to disseminate and poor clinical prognosis. It is well known that the development of any immune response requires time. However, the SCLC patients might not have any time to mount an appropriate immune response. Therefore, the timing of immunotherapy in relation to other therapies, in particular chemotherapy, is critical to allowing disease control and the development of an immune response. 
The aim of immunotherapy is to enhance the immune system's ability to recognize and specifically eliminate cancer cells while minimally impacting healthy lung tissue. There is mounting evidence that advanced immunotherapy is less effective than chemotherapy. However, the immunotherapy response is not significant or durable in SCLC. Evidence of an immune response in SCLC and a better understanding of the immunosuppressive tumor environment support the combined use of immunemodulators, such as Ipilimumab, with traditional chemotherapy regimens to improve patient outcomes and potentially sustain the effect from chemotherapeutic induction. Numerous findings support the clinical evaluation of different treatment schedules of immunotherapy with chemoradiotherapy or chemotherapy alone as strategies to circumvent the challenges associated with immunotherapeutic intervention for SCLC. Overcoming immune tolerance and improving the activation of antitumor $\mathrm{T}$ cells via combinatorial approaches could represent a new and more promising therapeutic application for active immunotherapies in SCLC.

Acknowledgments: This study was supported by the Natural Science Foundation of Jiangsu Province, China (grant no. BK20140736). We apologize to all researchers whose relevant contributions were not cited due to space limitations.

\section{References}

[1] SIEGEL RL, MILLER KD, JEMAL A. Cancer statistics, 2015. CA Cancer J Clin. 2015, 65: 5-29. http://dx.doi.org/10.3322/ caac. 21254

[2] SLOTMAN BJ, VAN TINTEREN H. Which patients with extensive stage small-cell lung cancer should and should not receive thoracic radiotherapy? Translational lung cancer research. 2015, 4: 292-4.

[3] KALEMKERIAN GP, AKERLEY W, BOGNER P, BORGHAEI H, CHOW LQ, DOWNEY RJ, et al. Small cell lung cancer. Journal of the National Comprehensive Cancer Network. 2013, 11: 78-98.

[4] KALEMKERIAN GP, GADGEEL SM. Modern staging of small cell lung cancer. Journal of the National Comprehensive Cancer Network. 2013, 11: 99-104.

[5] SPIGEL DR, TOWNLEY PM, WATERHOUSE DM, FANG L, ADIGUZEL I, et al. Randomized phase II study of bevacizumab in combination with chemotherapy in previously untreated extensive-stage small-cell lung cancer: results from the SALUTE trial. Journal of clinical oncology. 2011, 29: 2215-22. http://dx.doi.org/10.1200/JCO.2010.29.3423

[6] JOTTE R, CONKLING P, REYNOLDS C, GALSKY MD, KLEIN L, et al. Randomized phase II trial of single-agent amrubicin or topotecan as second-line treatment in patients with small-cell lung cancer sensitive to first-line platinumbased chemotherapy. Journal of clinical oncology. 2011, 29: 287-93. http://dx.doi.org/10.1200/JCO.2010.29.8851

[7] ARCARO A. Targeted therapies for small cell lung cancer: Where do we stand? critical reviews in oncology hematology. 2015, 95: 154-64.
[8] SPIGEL DR, GRECO FA, RUBIN MS, SHIPLEY D, THOMPSON DS, et al. Phase II study of maintenance sunitinib following irinotecan and carboplatin as first-line treatment for patients with extensive-stage small-cell lung cancer. Lung cancer. 2012, 77: 359-64. http://dx.doi.org/10.1016/j. lungcan.2012.03.009

[9] SPIGEL DR, SOCINSKI MA. Rationale for chemotherapy, immunotherapy, and checkpoint blockade in SCLC: beyond traditional treatment approaches. Journal of thoracic oncology. 2013, 8: 587-98. http://dx.doi.org/10.1097/ ITO.0b013e318286cf88

[10] MADDISON P, NEWSOM-DAVIS J, MILLS KR, SOUHAMI RL. Favourable prognosis in Lambert-Eaton myasthenic syndrome and small-cell lung carcinoma. Lancet. 1999, 353 117-8. http://dx.doi.org/10.1016/S0140-6736(05)76153-5

[11] WANG W, HODKINSON P, MCLAREN F, MACKINNON A, WALLACE W, et al. Small cell lung cancer tumour cells induce regulatory $\mathrm{T}$ lymphocytes, and patient survival correlates negatively with FOXP3+ cells in tumour infiltrate. international journal of cancer. 2012, 131: E928-37.

[12] TANI T, TANAKA K, IDEZUKA J, NISHIZAWA M. Regulatory $\mathrm{T}$ cells in paraneoplastic neurological syndromes. Journal of neuroimmunology. 2008, 196: 166-9. http://dx.doi. org/10.1016/j.jneuroim.2008.03.002

[13] KOYAMA K, KAGAMU H, MIURA S, HIURA T, MIYABAYASHI T, et al. Reciprocal CD4+ T-cell balance of effector CD62Llow CD4+ and CD62LhighCD25+ CD4+ regulatory $\mathrm{T}$ cells in small cell lung cancer reflects disease stage. Clinical cancer research. 2008, 14: 6770-9. http://dx.doi. org/10.1158/1078-0432.CCR-08-1156

[14] MELLMAN I, COUKOS G, DRANOFF G. Cancer immunotherapy comes of age. Nature. 2011, 480: 480-9. http://dx.doi. org/10.1038/nature10673

[15] ASMAR R, RIZVI NA. Immunotherapy for Advanced Lung Cancer. Cancer journal. 2015, 21: 383-91. http://dx.doi. org/10.1097/PPO.0000000000000151

[16] PARDOLL DM. The blockade of immune checkpoints in cancer immunotherapy. Nature reviews Cancer. 2012, 12: 252-64. http://dx.doi.org/10.1038/nrc3239

[17] YING CHENG,ET AL. Distribution and clinical significance of CTLA4, PD-1 and PD-L1 in peripheral blood of patients with small-cell lung cancer. 2015 ASCO Abstract 7574.

[18] MAYOR M, YANG N, STERMAN D, JONES DR, ADUSUMILLI PS. Immunotherapy for non-small cell lung cancer: current concepts and clinical trials. European journal of cardio-thoracic surgery. 2015. http://dx.doi.org/10.1093/ejcts/ $\underline{\text { ezv371 }}$

[19] TOPALIAN SL, HODI FS, BRAHMER JR, GETTINGER SN, SMITH DC, et al. Safety, activity, and immune correlates of anti-PD-1 antibody in cancer. The New England journal of medicine. 2012, 366: 2443-54. http://dx.doi.org/10.1056/ NEJMoa1200690

[20] DAVID R. Spigel, Karen L,et al. A Phase III Study(CheckMate 017) of Nivolumab (Anti-Programmed Death-1) vs Docetaxel in Previously Treated Advanced or Metastatic Squamous (SQ) Cell Non-Small-Cell Lung Cancer(NSCLC). 2015 ASCO Abstract 8009. 
[21] LUIS PAZ-ARES, et al. Phase III, Randomized Trial (CheckMate 057) of Nivolumab versus Docetaxel in Advanced Non-squamous (non-SQ) Cell Non-small Cell Lung Cancer (NSCLC). 2015 ASCO LAB109.

[22] YAMANE H, ISOZAKI H, TAKEYAMA M, OCHI N, KUDO $\mathrm{K}$, et al. Programmed cell death protein 1 and programmed death-ligand 1 are expressed on the surface of some small-cell lung cancer lines. American journal of cancer research. 2015, 5: $1553-7$.

[23] ISHII H, AZUMA K, KAWAHARA A, YAMADA K, IMAMURA Y, TOKITO T, et al. Significance of programmed cell death-ligand 1 expression and its association with survival in patients with small cell lung cancer. Journal of thoracic oncology. 2015, 10: 426-30. http://dx.doi.org/10.1097/ JTO.0000000000000414

[24] ANTONIA SCOTT, et al. Phase I/II Study (CheckMate 032) of Nivolumab With or Without Ipilimumab for Treatment of Recurrent Small Cell Lung Cancer (SCLC). 2015 ASCO Abstract 7503

[25] PATRICK A OTT, et al. Pembrolizumab (MK3475) in Patients With Extensive-Stage Small Cell Lung Cancer: Preliminary Safety And Efficacy Results From Keynote-028. 2015 ASCO Abstract 7502

[26] COLLINS AV, BRODIE DW, GILBERT RJ, IABONI A, MANSO-SANCHO R, et al. The interaction properties of costimulatory molecules revisited. Immunity. 2002, 17: 201-10. http://dx.doi.org/10.1016/S1074-7613(02)00362-X

[27] BUSTAMANTE ALVAREZ JG, GONZALEZ-CAO M, KARACHALIOU N, SANTARPIA M, VITERI S, TEIXIDO C, et al. Advances in immunotherapy for treatment of lung cancer. Cancer biology \& medicine. 2015, 12: 209-22.

[28] RECK M, BONDARENKO I, LUFT A, SERWATOWSKI P, BARLESI F, CHACKO R, et al. Ipilimumab in combination with paclitaxel and carboplatin as first-line therapy in extensive-disease-small-cell lung cancer: results from a randomized, double-blind, multicenter phase 2 trial. Annals of oncology. 2013, 24: 75-83. http://dx.doi.org/10.1093/annonc/mds213

[29] NOGUCHI A, KANEKO T, NAITOH K, SAITO M, IWAI $\mathrm{K}$, et al. Impaired and imbalanced cellular immunological status assessed in advanced cancer patients and restoration of the $\mathrm{T}$ cell immune status by adoptive T-cell immunotherapy. International immunopharmacology. 2014, 18: 90-7. http:// dx.doi.org/10.1016/j.intimp.2013.11.009

[30] ZHANG GQ, LI F, SUN SJ, HU Y, WANG G, et al. Adoptive immunotherapy for small cell lung cancer by expanded activated autologous lymphocytes: a retrospective clinical analysis. Asian Pacific journal of cancer prevention : APJCP. 2015, 16: 1487-94.

[31] DING X, CAO H, CHEN X, JIN H, LIU Z, WANG G, et al. Cellular immunotherapy as maintenance therapy prolongs the survival of the patients with small cell lung cancer. Journal of translational medicine. 2015, 13: 158. http://dx.doi. org/10.1186/s12967-015-0514-0

[32] FREEMAN-KELLER M, GOLDMAN J, GRAY J. Vaccine immunotherapy in lung cancer: Clinical experience and future directions. Pharmacology \& therapeutics. 2015, 153: 1-9. http://dx.doi.org/10.1016/i.pharmthera.2015.05.004
[33] SUZUKI H, OWADA Y, WATANABE Y, INOUE T, FUKUHARAV M, et al. Recent advances in immunotherapy for non-small-cell lung cancer. Human vaccines \& immunotherapeutics. 2014, 10: 352-7. http://dx.doi.org/10.4161/hv.26919

[34] GRANT SC, KRIS MG, HOUGHTON AN, CHAPMAN $\mathrm{PB}$. Long survival of patients with small cell lung cancer after adjuvant treatment with the anti-idiotypic antibody BEC2 plus Bacillus Calmette-Guerin. Clinical cancer research. 1999, 5: 1319-23.

[35] GIACCONE G, DEBRUYNE C, FELIP E, CHAPMAN PB, GRANT SC, MILLWARD M, et al. Phase III study of adjuvant vaccination with Bec2/bacille Calmette-Guerin in responding patients with limited-disease small-cell lung cancer (European Organisation for Research and Treatment of Cancer 08971-08971B; Silva Study). Journal of clinical oncology. 2005, 23: 6854-64. http://dx.doi.org/10.1200/JCO.2005.17.186

[36] CHADA S, MHASHILKAR A, ROTH JA, GABRILOVICH D. Development of vaccines against self-antigens: the p53 paradigm. Current opinion in drug discovery \& development. 2003, 6: 169-73.

[37] ANTONIA SJ, MIRZA N, FRICKE I, CHIAPPORI A, THOMPSON P, WILLIAMS N, et al. Combination of p53 cancer vaccine with chemotherapy in patients with extensive stage small cell lung cancer. Clinical cancer research. 2006, 12: 878-87. http://dx.doi.org/10.1158/1078-0432.CCR-05-2013

[38] CHIAPPORI AA, SOLIMAN H, JANSSEN WE, ANTONIA SJ, GABRILOVICH DI. INGN-225: a dendritic cell-based p53 vaccine (Ad.p53-DC) in small cell lung cancer: observed association between immune response and enhanced chemotherapy effect. Expert opinion on biological therapy. 2010, 10: 983-91. http://dx.doi.org/10.1517/14712598.2010.484801

[39] YANG L. TGFbeta, a potent regulator of tumor microenvironment and host immune response, implication for therapy. Current molecular medicine. 2010, 10: 374-80. http://dx.doi. org/10.2174/156652410791317039

[40] TIAN T, GU X, ZHANG B, LIU Y, YUAN C, et al. Increased circulating CD14(+)HLA-DR-/low myeloid-derived suppressor cells are associated with poor prognosis in patients with small-cell lung cancer. Cancer biomarkers. 2015, 15: 425-32. http://dx.doi.org/10.3233/CBM-150473

[41] ICLOZAN C, ANTONIA S, CHIAPPORI A, CHEN DT, GABRILOVICH D. Therapeutic regulation of myeloid-derived suppressor cells and immune response to cancer vaccine in patients with extensive stage small cell lung cancer. Cancer immunology immunotherapy. 2013, 62: 909-18. http://dx.doi. org/10.1007/s00262-013-1396-8

[42] THOMAS A, GIACCONE G. Why has active immunotherapy not worked in lung cancer? Annals of oncology. 2015, 26: 2213-20. http://dx.doi.org/10.1093/annonc/mdv323

[43] RAVI SALGIA, et al.Evaluation of CXCR4 expression on tumor and circulating tumor cells (CTCs) as predictive response marker for CXCR4 antagonist LY2510924 in combination with carboplatin-etoposide in extensive-disease small cell lung cancer (ED-SCLC). 2015 ASCO Abstract 7567.

[44] DOYLE A, MARTIN WJ, FUNA K, GAZDAR A, CARNEY D, et al. Markedly decreased expression of class I histocompatibility antigens, protein, and mRNA in human small-cell 
lung cancer. The Journal of experimental medicine. 1985, 161: 1135-51. http://dx.doi.org/10.1084/jem.161.5.1135

[45] PREDINA J, ERUSLANOV E, JUDY B, KAPOOR V, CHENG G, WANG LC, et al. Changes in the local tumor microenvironment in recurrent cancers may explain the failure of vaccines after surgery. Proceedings of the National Academy of Sciences of the United States of America. 2013, 110: E415-24. http:// dx.doi.org/10.1073/pnas.1211850110
[46] RUEGG C, PETERS S. Thalidomide in small cell lung cancer: wrong drug or wrong disease? Journal of the National Cancer Institute. 2009, 101: 1034-5. http://dx.doi.org/10.1093/jnci/ djp208

[47] FORMENTI SC, DEMARIA S. Combining radiotherapy and cancer immunotherapy: a paradigm shift. Journal of the National Cancer Institute. 2013, 105: 256-65. http://dx.doi. org/10.1093/jnci/djs629 